\title{
Simulation of Pulsed High Voltage DC Source And Blumlein Transmission Line
}

\author{
Mukhtar Hussain*, Tayyab Imran \\ Department of Physics \& Astronomy, College of Science, King Saud University, 11541 Riyadh, Saudi Arabia \\ *awanchep@gmail.com
}

\begin{abstract}
In the reported article, we have simulated the pulsed high voltage direct current (DC) source and Blumlein transmission line by Multisimulation13 (National Instruments, Inc). In the generation of high voltage pulses at the optimum repetition rate, we have observed and investigated the waveform behavior of voltage in the Blumlein transmission line.We present the simulation results of pulsed high voltage DC source with theoutput of $55.4 \mathrm{kV}$, operating at $131 \mathrm{~Hz}$.Further, voltage waveform across the Blumlein transmission line and its various parameters such as peak to peak voltage, root mean square voltage, peak to peak current, root mean square current and rate of oscillation of Blumlein transmission line are computed. The simulation of pulsed DC source and Blumlein transmission line by Multisimulation assisted in the designing and characterization of thehands-on high voltage source and Blumlein transmission line based transversely electrical excited at atmospheric (TEA) nitrogen laser.
\end{abstract}

Keywords: High Voltage, Pulsed DC source, Blumlein transmission line, Multisimulation

\section{Introduction}

At atmospheric pressure, the coherent ultraviolet light pulsesgenerated from the Blumlein transmission linebased transversely electrically excited nitrogen laser by employing pulsed DC high voltage as a pump source.The UV nitrogen laser pulses generated from the Blumlein transmission line and charge transfer circuit haveused in different applications. Such as the measurement of the speed of sound in various materials [1], observance of nanosecond scale plasma [2],bio-molecules analysis by nanoparticles based mass spectrometry [3], and surface-assisted laser desorption [4]. Further applications, thinfilm deposition for non-linear optical purposes [5],Te-doped Ge-Se thin films crystallization [6], andcategorization of change in phase of chalcogenide thin films [7].

The development and optimization of TEA nitrogen laser based on the Blumlein circuit [8], time-dependent inductance and resistance of free running spark gap of Blumlein based nitrogen laser for various resistive phase periods described $[9,10]$. The oscillatory behavior of power, current and the voltage across the laser discharge channel has observed [9]. The properties of discharge evaluation of Blumlein based pulsed laser described [11],and multiple switch Blumlein generator [12]. Theparametric analysis of Blumlein line nitrogen laser [13], for atmospheric pressure plasma treatment [14] nanosecond Blumlein based pulse source analysis [15] and Blumlein based nitrogen laser developed and investigated [8, 16-19].The Modeling of the Blumlein circuit and laser plasa resistance and inductance described [20].

A high voltage pumping source is required to generate ultraviolet coherent light pulses at atmospheric pressure. The coherent ultraviolet pulses were generated from the air at atmospheric pressure by employing DC high voltage source from the Blumlein transmission line electrical circuit [8]. The purpose of our present study is to simulate and optimize the pulsed DC high voltage source and the observance ofvariation of voltage waveform across the Blumlein transmission line. The electric discharge across the whole transmission lineresults in the emission of coherent UV pulses. Therefore, thebehavior of an oscillatory voltage needs to visualize across the Blumlein transmission line whichobserved by using a Multisimulationsoftware. Multisimulation is user-friendlyelectronics simulation software which can simulatevarious electronic circuits as well as engender prototype for printed circuit boards.

\section{Simulation of Pulsed De High Voltage Source}

The schematic setup of pulse DC, high voltage source,is shown in Fig. 1, which consist of three parts, regulated low voltage source output (12-15) V, LM555 CM based driver circuit whose ends connected to the flyback transformer which results pulsed high voltage signal. To generate high voltage pulsed DC source, Multisimulation is employed to simulatethe high voltage direct current, the high voltage DC source the circuit first which shown in Fig. 2. The output of 1B4B42 diodes based bridge rectifier is interconnected to MC7805CT voltage regulator along with $5 \mathrm{~V}$ source whose output fed into the LMC555CM based driver circuit.The driver circuit consists of 555 Timer integrated circuit (IC) along with the combination of resistors and capacitors which 
can generate the voltage pulses of various frequency. The range of frequecnises of the drive circuit varies from 5 $\mathrm{kHz}$ to $50 \mathrm{kHz}$. When the Flyback transformer is tuned at low frequencies, the output voltage pulses across Flyback transformer ends are low or vice versa.

The output pulses of 555 TimerIC further directed to the NPN transistor which acts as a fast switch and generates pulses in the nanosecond range. Finally, the output of transistor and regulator used as input to flyback transformer which eventually results in high voltage direct current pulses.
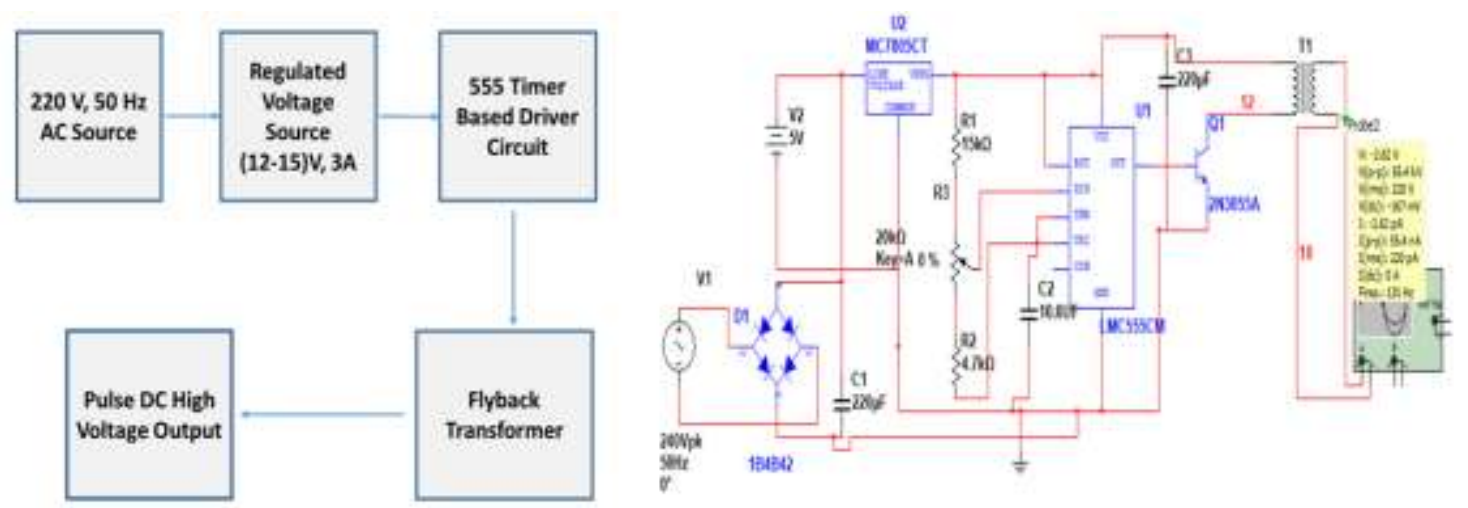

Fig. 1 Schematic of high voltage power Fig. 2 Circuit diagram of pulsed de high voltage source

The Blumlein transmission line consists of parallel plate capacitor interconnected by an inductor as shown inFig. 3. The electric field estimation across the Blumlein transmission line is necessary to monitor the lifetime ofits operation particularly when it employed to fabricate a laser system which can be obtained by the simulation of Blumlein circuit in Multisimulation. The behavior of the voltage waveform across the Blumlein transmission line determined its efficiency and performance which is optimized and observed by Multisumlation.

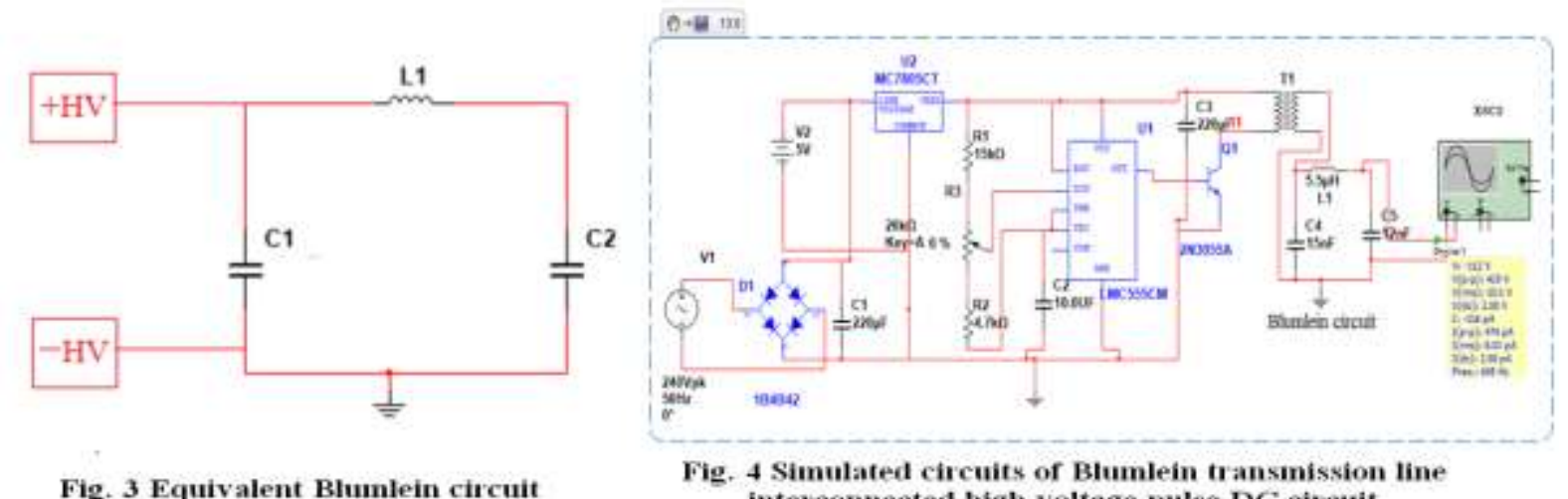

Fig. 3 Equivalent Blumlein circuit

Fig. 4 Simulated circuits of Blumlein transmission line interconnected high voltage pulse DC circuit

The simulated diagram of Blumlein transmission line interconnected with the pulsed DC high voltage source shown in Fig. 4. The Blumlein transmission line has two parallel capacitors of capacitance $15 \mathrm{nF}$ and 12 $\mathrm{nF}$ respectively connected through $5.5 \mu \mathrm{H}$ inductor. When the pulsed DC source applied across the ends of Blumlein transmission line, it behaves as an LC oscillator, which generates the electromagnetic pulses. In the Blumlein circuit, electric dischargecreated across the Blumlein transmission line by employing high voltage direct current source. The oscillatory voltage behavior visualized and computedto observe the reliable and stable performance across the Blumlein transmission line todevelop Blumlein based laser source, multiple switch generator, and Blumlein based nanosecond pulse generator.

\section{Results And Discussion}

The output waveform of simulated pulsed DC high voltage source shown in Fig. 5 which observed by usingmultichannel oscilloscope. The maximum computed peak to peak value of voltage by probe measurement is $55.4 \mathrm{kV}$ as shown in Fig. 2 is quite consistent with the measured results as illustrated in Fig. 5.The repetition rate of the pulsed dc high voltage source is computed to be $\sim 131 \mathrm{~Hz}$ with a $55.4 \mathrm{nA}$ peak to peak current as shown in Fig. 3 by the probe results. 


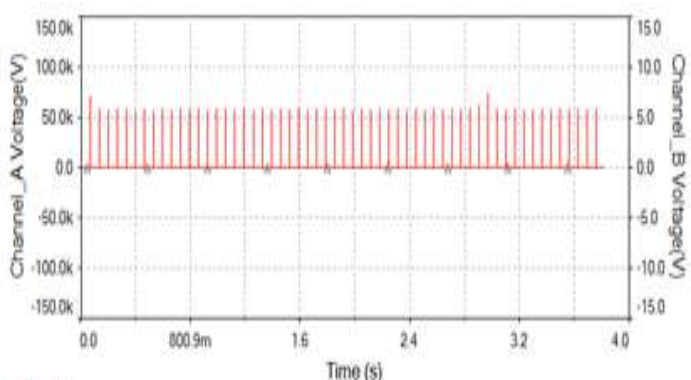

$10 \frac{0 \mathrm{men}}{2}$

Figure 5 Voltage waveform of Pulsed DC High Voltage source

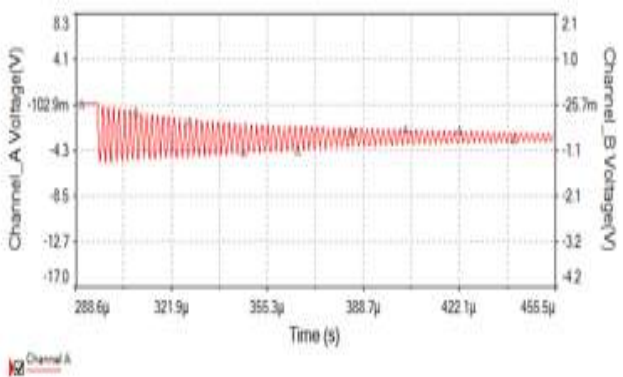

Figure 6 Oscillatory waveform computed for $0.5 \mathrm{~ns}$

The voltage waveform variation across the Blumlein transmission line for a time interval of $0.5 \mathrm{~ns}$ and $1.5 \mathrm{~ms}$ computed as shown inFig. 6 andFig. 7. There is an oscillatory behavior of voltage whose amplitude falls within a very short period which generates the electromagnetic pulses or coherent ultraviolet pulse be contingent to geometry. The detailed observation of voltage waveform is clearer and concise in Fig. 8 and Fig. 9, which evidently portrays thecomportment of the voltage across the Blumlein transmission line for $0.5 \mathrm{~ns}$. The behavior of simulated voltage across the Blumlein transmission line validated the numerically reported results [9, 13].

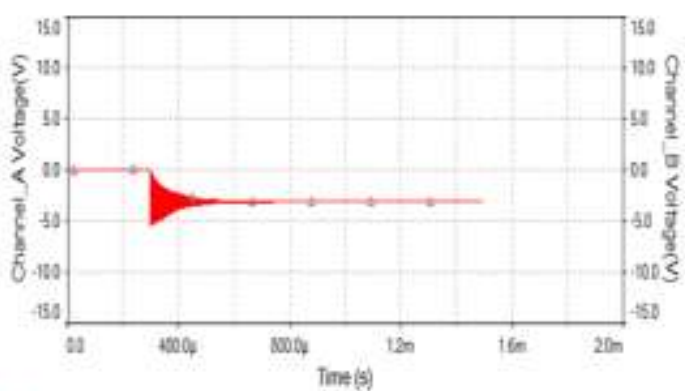

$12^{0}=14$

Fig. 7 Oscillatory behavior of voltage in the Blumlein transmission line for time-period of 1 ms

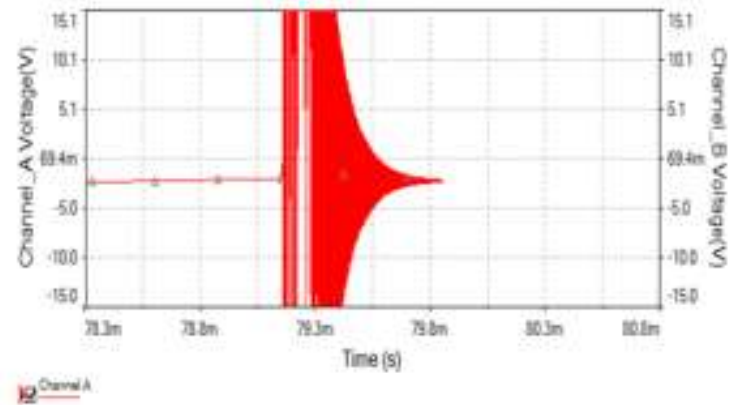

Fig. 8 Variation of the voltage waveform in the Blumlein transmission line for time-period of $80 \mathrm{~ms}$

The description of voltage waveform after $80.0 \mathrm{~ms}$ shown in Fig.8. The behavior of the voltage in the Blumlein transmission is similar as that for $0.5 \mathrm{~ns}$ and $1.0 \mathrm{~ms}$. The detailed behavior of the whole Blumlein transmission line voltage waveform shown in Fig.9which depicts theoscillatory waveform of voltage in Blumlein transmission. The amplitude decreases and falls to zero within $0.5 \mathrm{~ms}$; then maximum amplitude achieved after $6.5 \mathrm{~ms}$ which falls again to zero after $0.5 \mathrm{~ms}$ as shown in Fig.9. At the highestamplitudeof voltage waveform, the potential difference across the transmission line is maximum, consequently strong electric fieldcreated across the Blumlein transmission line.Subsequently, when the amplitude falls, the strength of electric field decreases across the Blumlein transmission line, so as potential difference as well. Such behavior of voltage in the Blumlein transmission line practically generates the coherent ultraviolet pulses in the air in the form of TEA Nitrogen Laser as reported [8-11,13].

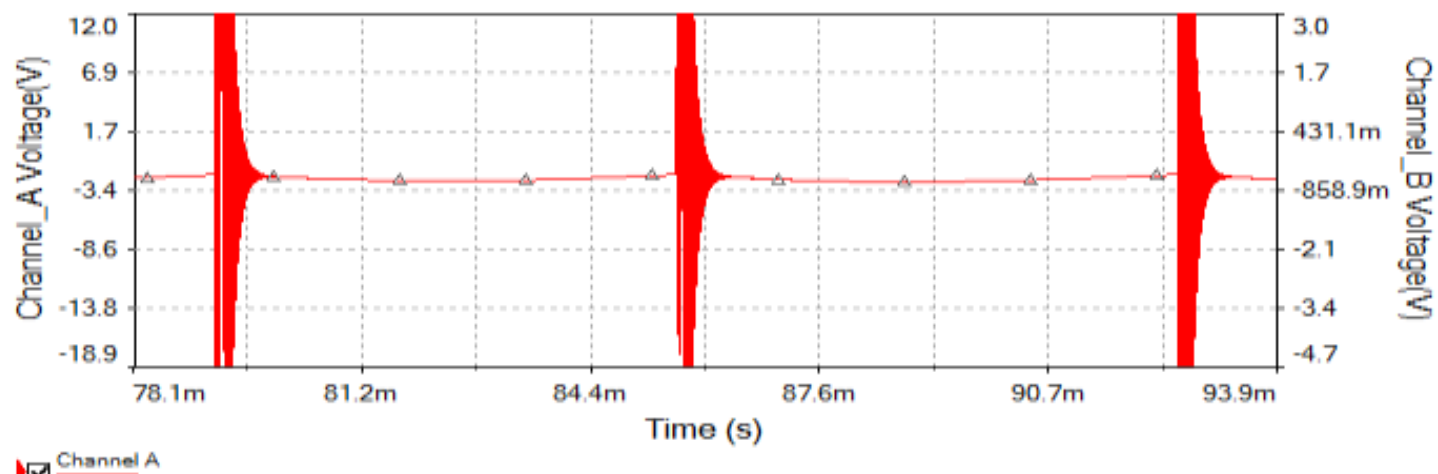

Fig. 9 Oscillatory behavior of voltage in the Blumlein transmission line 
The simulated voltage waveform variation across the Blumlein transmission lineshown in Fig. (6-9) have the consistency with the computational model of Blumlein line nitrogen laser $[9,13]$. Such behavior of voltage variation across the Blumlein circuit generates thecoherent ultraviolet pulsesat the atmospheric pressure as well as in vacuum by employing reported high voltage pulse DC source as a pump source.

\section{Conclusion}

The simulation of Blumlein transmission line and pulsed DC high voltage source by using Multisimulation software described and optimized. The pulse DC voltage source with output $55.4 \mathrm{kV}$ obtained at the repetition rate of $131 \mathrm{~Hz}$. The oscillatory behavior of voltage in the Blumlein transmission line is computed and observed which generates the coherent ultraviolet pulses centered at $337.1 \mathrm{~nm}$. The maximum amplitude of the oscillatory wave achieved after $6.5 \mathrm{~ms}$ which falls to zero after $0.5 \mathrm{~ms}$ in the Blumlein transmission line and similar behavior continue which generates the electromagnetic pulses or coherent ultraviolet pulses. The voltage waveform across the Blumlein transmission line and its various parameters such as peak to peak voltage, root mean square voltage, peak to peak current, root mean square current and rate of oscillation of transmission line are computed and presented, for the reliable and consistent operation of Blumlein line base laser source or pulse generator.

\section{Acknowledgements}

The authors acknowledge the financial support from the King Abdulaziz City for Science and Technology (KACST) under the project number M S-36-2.

\section{References}

[1] A.S. Provorov, V.V. Salmin, A.B.Salmina, A.A. Fursov, A.V. Stepanenko, A.G. Sokolovich, and R.Y. Olovyannikova, Pulsed gas lasers with longitudinal discharge and their application in medicine, Laser physics, 15(9), 2005, 1299-1302.

[2] M.S. Averin, O.A. Bashutin, E.D. Vovchenko, L. San Wei, D.E. Prokhorovich, A.S. Savjolov, S.A. Savjolov, A multichannel TEA N2 laser for visualizing pulsed plasma in the nanosecond range, Instruments and Experimental Techniques, 47 (2),2004, $209-213$.

[3] C.K. Chiang, W.T. Chen, and H.T. Chang, Nanoparticle-based mass spectrometry for the analysis of biomolecules, Chemical Society Reviews, 40 (3), 2011, 1269-1281.

[4] T. C. Chiu, L.C. Chang, C.K. Chiang, and H.T. Chang, Determining estrogens using surface-assisted laser desorption/ionization mass spectrometry with silver nanoparticles as the matrix, Journal of the American Society for Mass Spectrometry, 19(9), 2008,1343-1346.

[5] H. El Ouazzani, S. Dabos-Seignon, D. Gindre, K. Iliopoulos, M. Todorova, R. Bakalska,and B. Sahraoui, Novel styrylquinolinium dye thin films deposited by pulsed laser deposition for nonlinear optical applications, The Journal of Physical Chemistry C, 116(12), 2012, 7144-7152.

[6] F.A. Al-Agel, Structural and optical properties of Te doped Ge-Se phase-change thin films: a material for optical storage, Materials Science in Semiconductor Processing, 18, 2014, 36-41.

[7] M.A. Alvi, M. Zulfequar, and A.A. Al-Ghamdi, Characterization of phase change Ga 15 Se 77 Ag 8 chalcogenide thin films by laser-irradiation, Journal of Alloys and Compounds, 550, 2013,431-437.

[8] M. Hussain and T. Imran, Design and construction of prototype transversely excited atmospheric (TEA) nitrogen laser energized by a high voltage electrical discharge, Journal of King Saud University-Science, 27(3), 2015, 233-238.

[9] M. Twati,A full distributed parameter model of a Blumlein-line laser circuit including the effect of time varying spark-gap inductance and resistance", Electrical and Computer Engineering (CCECE), IEEE 27th Canadian Conference on. IEEE, 2014.

[10] M. Hussain, M. B. Siddique and T. Imran, Analysis of transversely electrical excited atmospheric (TEA) nitrogen laser and different parameters of homemade ignition system, Science International, 27(6),2015, 5001-5004.

[11] S. B. Han and S. H. Park, Evaluation of Discharge Characteristics Followed by the Development of Blumlein pulsed power source, Journal of the Korean Institute of Illuminating and Electrical Installation Engineers, 24(10),2010, 99-105.

[12] Z. Liu, K. Van, G. J. J. Winands, E. J. M. Van Heesch, and A. J. M. Pemen, Novel multiple-switch Blumlein generator, Review of scientific instruments, 77(3),2006,033502.

[13] M. O Twati, and A. B. Otman, Distributed parameter analysis of a Blumlein-line N2 laser, Optics communications, 99(5-6), 1993, 405-412.

[14] N. Nayan, M. R. Zahariman, M. F. B. Ahmad, R. A. M. Ali, M. Z. Sahdan, and U. Hashim,Development and application of inhouse high voltage power supply for atmospheric pressure plasma treatment system,In Semiconductor Electronics (ICSE), 10th IEEE International Conference on, 2012, 596-599.

[15] Y.S. Roh, and Y.S. Jin,Analysis of output pulse of high voltage and nanosecond Blumlein pulse generator, Journal of Electrical Engineering and Technology, 8(1), 2013, 150-155.

[16] W. Fitzsimmons, L. Anderson, C. Riedhauser, and J. Vrtilek, Experimental and theoretical investigation of the nitrogen laser, IEEE journal of quantum electronics, 12(10), 1976, 624-633.

[17] J. D. Shipman Jr, Traveling wave excitation of high power gas lasers, Applied Physics Letters, 10(1), 196, 3-4.

[18] J. G. Small, and R. A.Ashari, A Simple Pulsed Nitrogen 3371 A Laser with a Modified Blumlein Excitation Method, Review of Scientific Instruments, 43( 8),1972, 1205-1206.

[19] C. Iwasaki, and T. Jitsuno,An investigation of the effects of the discharge parameters on the performance of a TEA N 2 laser, IEEE Journal of Quantum Electronics, 18(3),1982,423-427.

[20] C. Zhao, J. Wu. Modeling of Blumlein Circuit and Calculation of Resistance and Inductance of Laser Plasma. International Journal of Science, Technology and Society. Vol. 2, No. 6, 2014, pp. 196-200. doi: 10.11648/j.ijsts.20140206.16 